\title{
Spatial attention is necessary for object-based attention: Evidence from temporal-order judgments
}

\author{
Ian Donovan $^{1} \cdot$ Jay $_{\text {Pratt }^{2}} \cdot$ Sarah Shomstein $^{3}$
}

Published online: 27 December 2016

(C) The Psychonomic Society, Inc. 2016

\begin{abstract}
Attentional selection is a dynamic process that relies on multiple types of representations. That object representations contribute to attentional selection has been known for decades; however, most evidence for this contribution has been gleaned from studies that have relied on various forms of spatial cueing (some endogenous and some exogenous). It has thus remained unclear whether object-based attentional selection is a direct result of spatial cuing, or whether it still emerges without any spatial marker. Here we used a novel method - the temporal-order judgment (TOJ) - to examine whether object-based guidance emerges in the absence of spatial cuing. Participants were presented with two rectangles oriented either horizontally or vertically. Following a 150 -ms preview time, two target stimuli were presented on the same or on different objects, and participants were asked to report which of the two stimuli had appeared first. The targets consisted of stimuli that formed a percept of a "hole" or a "hill." First, we demonstrated that the "hill" target was indeed processed faster, as evidenced by a positive perceived simultaneity (PSS) measure. We then demonstrated that if two targets appeared with equal probabilities on the same and on different objects, the PSS values, although positive, were not modulated by the objects. In a subsequent set of experiments, we showed that objects can modulate attentional allocation-
\end{abstract}

Ian Donovan

ian.donovan@nyu.edu

1 Department of Psychology, New York University, New York, NY, USA

2 Department of Psychology, University of Toronto, Toronto, Ontario, Canada

3 Department of Psychology, George Washington University, Washington, DC, USA however, only when they are biased by a spatial (endogenous) cue. In other words, in the absence of a spatial cue or bias, object representations do not guide attentional selection. In addition to providing new constraints for theories of objectbased attentional guidance, these experiments introduce a novel paradigm for measuring object-based attentional effects.

Keywords Object-based attention - Space-based attention . Temporal processing

To cope with the vast amount of incoming sensory information, the visual system must establish a stable representation of the environment and selectively process relevant information. Visual attention is a selection mechanism gating what and how visual information is processed, and given the retinotopic organization of the visual system, is largely mediated by spatial locations. Such inherent reliance on spatial coding has been demonstrated across numerous cuing studies. In most of these studies, spatial attention enhances performance: when a spatial location is cued, either by an exogenous sensory event or by endogenous instruction, targets are detected and identified more quickly and accurately if they appear near the cued location, relative to uncued locations (for reviews, see Carrasco, 2011; Eriksen \& Eriksen, 1974; Posner, 1980; Posner, Walker, Friedrich, \& Rafal, 1984).

In addition to the multitude of research elucidating the contribution of spatial information to attentional selection, a corresponding line of research has established that objects within a visual environment also guide selection (Chen, 2012; Duncan, 1984; Scholl, 2001), and that mechanisms for space-based and object-based attention coexist and dynamically contribute to selection in distinct ways (Egly, Driver, \& Rafal, 1994; Lavie \& Driver, 1996; Shomstein, 2012). Importantly, it is crucial to note that the vast majority of 
studies that have revealed the contribution of object representation to attentional guidance relied on spatial cuing paradigms either with an exogenous and/or endogenous cues or expectations (e.g., Arrington, Carr, Mayer, \& Rao, 2000; Becker \& Egeth, 2000; Chen \& Cave, 2008; Egly et al., 1994; Fiebelkorn, Saalmann, \& Kastner, 2013; Hollingworth, Maxcey-Richard, \& Vecera, 2012; Kravitz \& Behrmann, 2011; Pratt \& Sekuler, 2001; Vecera, 1997; Watson \& Kramer, 1999). As a result, it remains unclear whether object representations influence attentional selection only when attention is initially directed to a spatial region occupied by an object rather than by an external cue or internal bias.

A large body of evidence in support of object-based attention has been derived from variants of the two-rectangle paradigm first developed by Egly, Driver and Rafal (1994). In this paradigm, observers are instructed to maintain central fixation throughout each trial, and are presented with two adjacent rectangles, oriented either horizontally or vertically. After a brief delay, one end of one of the rectangles is briefly illuminated, exogenously cuing attention to that spatial location. Following another brief delay, a target stimulus appears within one of the rectangles, either in the same location as the cue (the valid cue location), on the same object but on the opposite end (the invalid same-object location), or on the other object (the invalid different-object location). It is generally observed that participants exhibit peak performance (fast reaction times and high accuracy) in the valid location, a finding that is taken as a marker of space-based attentional selection. Importantly, participants are significantly faster and more accurate to identify the target in the invalid same-object location than in the invalid different-object location, despite the fact that both are equidistant from the cue. The difference between the two invalid conditions is taken as a marker of an objectbased effect since object structure is the only apparent distinguishing feature between the two trial types. It should be noted that the exogenous cues used in many of these studies also provides probability information, with the cued location being the most likely target location (Egly et al., 1994), although uninformative exogenous cues have been shown to produce similar effects (for review see Chen, 2012; Chen \& Cave, 2006; Shomstein \& Yantis, 2002, 2004). Regardless of the probabilities involved, presence of spatial cues means that it is not possible, in and of itself, to determine the role of only the object on the allocation of attention.

Although the majority of studies demonstrating the contribution of object representations to attentional selection relied on spatial cuing, some studies report object-based guidance of attention in paradigms that do not use spatial cues. The most replicated finding is a flanker interference effect as a function of whether flankers appear on the same- or different object (Chen \& Cave, 2006; Driver \& Baylis, 1991; Kramer \& Jacobson, 1991; Richard, Lee, \& Vecera, 2008; Shomstein \& Yantis, 2002). Although this procedure and task eliminate reliance on the exogenous spatial cuing present in the tworectangle paradigm, it introduces a $100 \%$ predictable endogenous spatial cue. Targets in these flanker interference tasks always appear in the center of the display, and thus participants benefit from endogenously attending to this spatial location, where the target will certainly appear. Therefore, object-based attention studies that employ flanker interference tasks do not provide insight into whether object-based guidance of attentional selection emerges purely as a consequence of spatial cuing.

Given that both exogenous and endogenous cues generate spatial biases in the allocation of attention, and since the existing studies demonstrating object-based effects have cued or biased attention to particular spatial locations, it remains unclear whether visual objects guide attention in and of themselves. The goal of the present study, therefore, is to employ a paradigm that does not rely on an initial bias of a particular spatial location in order to examine the contribution of object representations to attentional guidance. Specifically, we adopt the temporal-order judgment (TOJ) method to assess visual prior entry, by presenting two competing targets within either the same object or two different objects.

Visual prior entry is a sensitive measure of initial attentional deployment, and provides a direct and accurate measure of the way attention is distributed in a scene. Prior entry, as an effect, is explained in terms of attentional selection because selection accelerates processing of sensory stimuli, thereby decreasing the time between the physical onset of a stimulus and its further processing (for reviews, see Shore, Spence, \& Klein, 2001; Spence \& Parise, 2010; Stelmach \& Herdman, 1991; Ulrich, 1987). In other words, a stimulus cannot be processed further by the visual system without the simultaneous involvement of both attentional and perceptual processes (e.g., Serences \& Yantis, 2006). Therefore, an attended stimulus will reach awareness before other competing stimuli. Typical TOJ tasks require participants to fixate on a central location in preparation for the onset of two target items that are separated by a variable interval. Participants report that item had the first onset at different stimulus onset asynchrony (SOA) intervals, producing a TOJ response function. A point of perceived stimulus simultaneity (PSS) is then calculated, indicating the interval needed for the participant to perceive both target items as presented simultaneously. Thus, the PSS represents how much time the unattended stimulus must occur before the attended stimulus, due to prior entry, for them to be perceived as occurring simultaneously. The TOJ method, through the PSS, provides a precise measure of the influence of attentional selection on the relative speed at which two competing stimuli are processed. Prior entry has been observed for stimuli that appear in a cued location (Spence, Shore, \& Klein, 2001), as well as stimuli that are more salient or motivationally relevant than competing targets (West, Anderson, Bedwell, \& Pratt, 2010; West, Anderson, \& Pratt, 2009). 
In the present study, we used a novel TOJ paradigm with a display modeled on the two-rectangle object-based attention paradigm. Specifically, we presented participants with two rectangles, oriented either horizontally or vertically, before the onset of two target items. Of the two target items, one was expected to elicit prior entry effects due to the visual features of the stimulus itself (i.e., a more salient stimulus) and the stimulus array. We assessed the degree of prior entry for this prioritized stimulus, using PSS as a metric. The target items appeared within either the same object (same-object) or two different objects (different-object), providing a dissociation for prior entry on same-object versus different-object trials. Importantly, our design provides, for the first time, a measure of the influence of visual objects on attentional guidance and selection without the use of an endogenous or exogenous spatial cue.

\section{Experiment 1}

As a first step, it is necessary to establish a paradigm and a stimulus set that reliably elicit prior-entry effects in the absence of space- or object-based cuing. In Experiment 1, we examined the extent to which the visual features of competing target stimuli affect the TOJs (i.e., the perception of which target appeared first) when the stimuli appear within a single, unified object (Fig. 1a). Specifically, we used a high-contrast background pattern consisting of black and white stripes, and two stimulus types (Fig. 1c and d), one that consisted of stripes matching the pattern exactly, creating a percept of a punch through (we will refer to this as a "hole"), and another that conflicted with the pattern (i.e., was rotated $45^{\circ}$; we will refer to this as the "hill"). Prior research has suggested that the "hill" stimulus elicits prior-entry effects, such that when presented with two stimuli simultaneously, participants perceive the "hill" as appearing before the "hole" (West, Pratt, \& Peterson, 2013). The primary goal for Experiment 1 was to establish that the stimulus set adopted for the present experimental procedure was capable of eliciting a prior-entry effect for the "hill."

\section{Method}

Participants Twenty-four undergraduates (15 women, nine men) from George Washington University participated in Experiment 1 for course credit. All participants were naive to the purpose of the study and had normal or corrected-tonormal vision.

Apparatus and procedure The stimulus set was displayed on a 15 -in. monitor at a resolution of $1,024 \times 768$ pixels, using a
Dell Optiplex 745. Participants were seated approximately $62 \mathrm{~cm}$ away from the monitor.

A schematic of a typical trial sequence is shown in Fig. 1a. Each trial began with a fixation display that consisted of a high-contrast background pattern of black stripes of alternating widths $\left(0.08^{\circ}\right.$ and $\left.0.14^{\circ}\right)$ separated by white stripes (average width of $0.10^{\circ}$, standard deviation of $0.04^{\circ}$ ). The stripes were oriented $48.86^{\circ}$ counterclockwise from horizontal, and the pattern was rendered on a black background. A black cross $\left(0.16^{\circ} \times 0.16^{\circ}\right)$ on top of a white square $\left(0.22^{\circ} \times 0.22^{\circ}\right)$ was positioned in the center of the patterned area. Participants were instructed to fixate on the cross for the duration of the experiment. After a variable interval $(500,600,700$, or $800 \mathrm{~ms})$, a white square $\left(2.13^{\circ} \times 2.13^{\circ}\right.$; Fig. 1a) with a cutout $\left(0.81^{\circ} \times\right.$ $\left.0.81^{\circ}\right)$ in the center appeared on top of the stripped pattern for $1,000 \mathrm{~ms}$. We refer to this configuration as the "cutout." On each trial, two target stimuli, circular shapes $0.43^{\circ}$ in diameter, appeared in two adjacent corners within the cutout $\left(0.14^{\circ}\right.$ from the nearest two edges, $1.06^{\circ}$ from center of fixation). One of the targets matched the background pattern (i.e., the "hole"), whereas the other consisted of a background pattern rotated $45^{\circ}$ clockwise (i.e., the "hill"). Two targets appeared with variable SOA, either simultaneously or within a short delay of one another $(0,12,24,36,48$, or $60 \mathrm{~ms})$. The delay and order of the preceding target (e.g., hill appearing first) was randomized, and each value was equally likely. The targets remained on screen for $62 \mathrm{~ms}$, followed by a black screen that remained until a response. Participants were asked to report the location of the stimulus that had appeared first, by using a keyboard key corresponding to the corner in which the stimulus had appeared (D for top left, $\mathrm{C}$ for bottom left, $\mathrm{K}$ for top right, and $\mathrm{M}$ for bottom right). Participants were encouraged to respond as accurately as possible. Response speed was deemphasized, since there was an unlimited amount of time to give a response. Participants were instructed to respond as accurately as possible. After a response was made, the next trial began immediately.

Design Participants completed 16 practice trials before beginning the experimental trials, which consisted of eight blocks of 88 trials each. On all trials, the hill and the hole were equally likely to appear first. The stimuli were equally likely to appear horizontally and vertically, relative to each other. Trial order was randomized across all factors (first onset stimulus, horizontal versus vertical stimulus orientation, and SOA). Short, self-paced breaks were given between blocks.

\section{Results and discussion}

Six participants were excluded from the analysis due to low cumulative accuracy (less than $70 \%$ correct). Additionally, all trials on which the keyed response did not correspond with the 


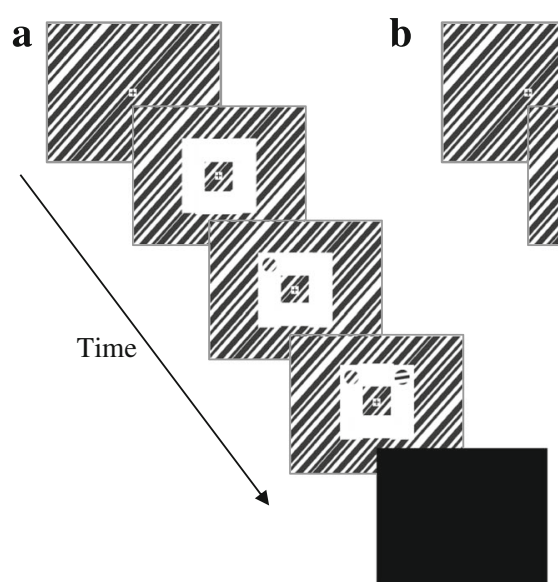

Fig. 1 Trial sequence and stimulus examples. (a) Trial sequence from Experiment 1, with targets appearing in a single white square cutout shape. (b) Trial sequence from Experiments 2, 3, and 4. Note that the trial sequence is identical to that in Experiment 1, except that two white rectangles of the same width and height appear on either side of fixation,

location of either of the targets were excluded from the analysis $(<3.2 \%$ of responses). For example, if a trial consisted of a top left and a bottom left stimuli, yet the participant responded with the key corresponding to the upper right, this trial was excluded from the analysis. Figure 2 shows the average proportions of responses on which participants indicated that a "hole" stimulus had appeared first ("hole" first), as a function of SOA. Positive SOAs refer to when the "hole" was presented first, whereas negative SOAs refer to trials on which the "hill" was presented first. A TOJ function was then derived by fitting the data to a sigmoidal function: $f(x)=y 0+\frac{a}{1+e^{-\left(\frac{x-x 0}{b}\right)}}$.

The effect of prior entry was assessed by calculating each participant's individual PSS values, indicating the interval needed for the participant to perceive both target items as

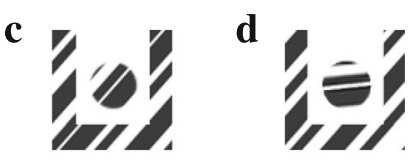

Until key-press

either horizontally or vertically oriented (vertical is shown). (c) Example of a "hole stimulus": A circular stimulus within the white rectangular object, with lines congruent with the background. (d) Example of a "hill stimulus," which is identical to the "hole" stimulus, but its lines are rotated clockwise, such that they are incongruent with the background

appearing simultaneously. This was derived by fitting the data for each participant to a sigmoid (see Fig. 2), and determining the value of $x$ (time in milliseconds) at which the value of $y$ (proportion of reporting that the "hole" is reported first) was equal to .5 , indicating the time at which the participant was equally likely to respond that "hill" or "hole" had appeared first. Positive values indicate that the "hole" would need to appear that many milliseconds before the "hill" for it to be perceived as appearing at the same time.

Participants completed Experiment 1 with an average accuracy of $83.52 \%$. The average PSS was $2.64 \mathrm{~ms}$, which was significantly different from zero $[t(17)=11.80, p<.001$, Cohen's $d=5.724$, scaled JZS Bayes factor $=8,102,745]$ (Fig. 2a). The positive PSS value indicates a prior-entry effect for the "hill." Notably, all participants showed PSS values

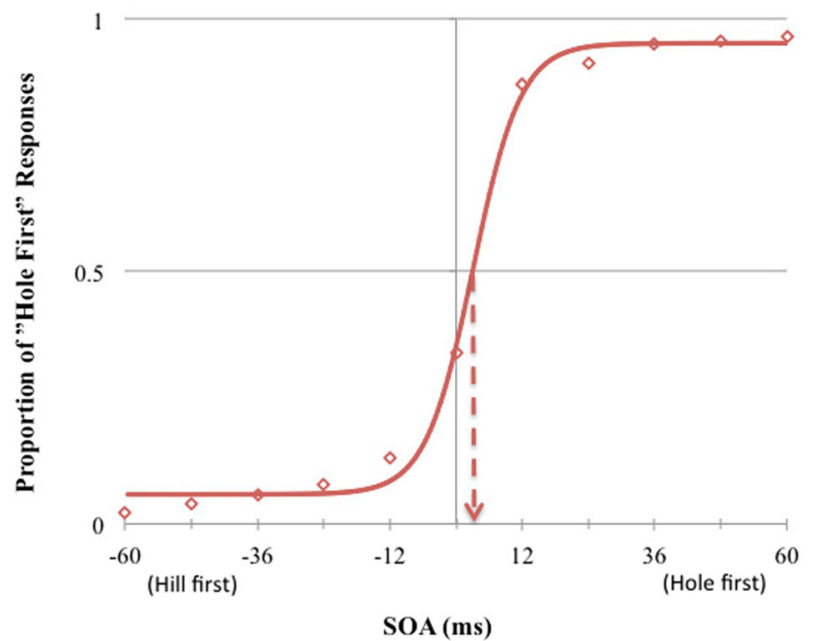

b

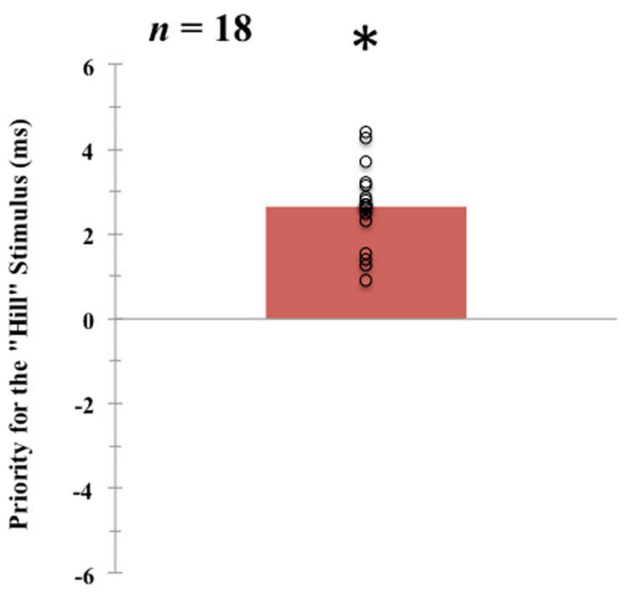

Fig. 2 Results from Experiment 1. (a) Aggregate temporal-order judgment (TOJ) function. (b) Average priority for the "hill" stimulus and individual results 
within two standard deviations of the mean (Fig. 2b). An analysis of variance (ANOVA) with Orientation of the Two Targets (top, bottom, left, and right) as a within-subjects factor revealed no significant effect on prior entry of the "hill," suggesting a lack of any contribution from a set of specific target locations.

Two major results emerged from this experiment. First, the "hill" stimulus was confirmed to exhibit prior entry over the "hole," indicating that the visual features of the "hill" stimulus relative to the background leads to faster detection of the "hill" than detection of the "hole." Second, PSS does not depend on target orientation as evidenced by horizontally and vertically oriented targets eliciting the same degree of prior entry. This result indicates that attention is distributed evenly across all target locations. Notably, our effect size was small, with an average PSS of $2.64 \mathrm{~ms}$ for the "hill," but with a high power (Cohen's $d=5.724$ ), we are confident that these results indicate reliable prior entry for the hill with this procedure.

\section{Experiment 2}

The results of Experiment 1 indicate that, in the absence of spatial cuing, when two stimuli appear in the same object, a stimulus that conflicts with a background pattern exhibits prior entry (i.e., is perceived faster) relative to a stimulus that matches the background pattern. To examine the influence of objects on prior-entry effects, we altered the stimulus set from Experiment 1 to include two parallel rectangles that were of the same thickness and positioning them as the sides of the "cutout" (Fig. 1b). Targets appeared in exactly the same absolute spatial locations as in Experiment 1, but now they either appeared on the same object or in two different objects. If object representations contribute to attentional guidance, then the magnitude of the prior-entry effect will be modulated by targets appearing on the same or on a different object, with reduced PSS magnitude for targets that appear on the same object. Alternatively, it may be that without spatial cues to bias attention to a particular object, object representations do not contribute to attentional guidance. If this is the case, then prior-entry effects will not be modulated by targets appearing on the same or on different objects.

\section{Method}

Participants Seventy-six undergraduates ${ }^{1}$ (61 women, 15 men) from George Washington University participated in return

\footnotetext{
${ }^{1}$ This sample size is larger than that in Experiment 1 and the subsequent experiments, due to an error in recruitment. The target number of participants was 20 per each object orientation (power analysis based on similar effect sizes). See the second-to-last paragraph in the Discussion section for the measures taken.
}

for course credit. All participants were naive to the purpose of the study and had normal or corrected-to-normal vision.

Apparatus, procedure, and design The apparatus, procedure, and design were the same as in Experiment 1, except that the cutout was replaced by two rectangles $\left(0.65^{\circ} \times 2.13^{\circ}\right)$, with a $0.81^{\circ}$ edge-to-edge separation between the rectangles (Fig. 1b). The rectangle orientation (horizontal or vertical) was varied between subjects and was determined randomly for each participant. Stimuli were equally likely to appear in the same and in different objects. Trial order was randomized across all factors (first onset stimulus, same or different object, and SOA).

\section{Results and discussion}

As in the previous experiment, participants with an overall accuracy less than $70 \%$ were excluded from the analysis. Responses that did not correspond to the two target locations were also excluded from the analysis $(<1.40 \%$ of responses). Because the focus of this experiment was on examining the influence of objects on prior entry, participants who did not demonstrate prior-entry effects were excluded from the analysis. Those whose PSS values exceeded two standard deviations from the mean for either same-object or differentobject trials were excluded (the respective means and standard deviations for same and different objects were calculated separately). In total, 24 participants were excluded from the analysis.

The average accuracy was $90.62 \%$ (excluding trials with an SOA of $0 \mathrm{~ms}$ ). The average accuracies for same-object and different-object trials were $91.34 \%$ and $89.91 \%$, respectively. Figure 3 a shows an aggregate TOJ function: sigmoids fitted to data averaged for observers and divided by same-object and different-object trials, with arrows indicating the aggregate PSS. Figure $3 \mathrm{~b}$ shows average PSSs in different-object trials and in same-object trials, with black circles indicating the individual values. Figure $3 \mathrm{c}$ shows the individual PSS values for both different-object trials ( $x$-axis) and same-object trials ( $y$-axis), with different colors indicating vertically oriented objects and horizontally oriented objects. The last plot shows a general trend toward positive PSS values on same-object trials, different-object trials, or both for the majority of participants. An ANOVA on PSS values with Object (different vs. same object) as a within-subjects factor and Object Orientation (vertical vs. horizontal) as a between-subjects factor revealed no main effect of object (different vs. same object) and no effect of object orientation (vertical vs. horizontal) on PSSs. The average PSS for different-object trials was $1.261 \mathrm{~ms}$, and that for same-object trials was $0.964 \mathrm{~ms}$ (collapsed over orientations), both of which were significantly different from 0 [different object: $t(51)=5.926, p<.001$, Cohen's $d=1.011$, scaled JZS Bayes factor $=55,992.47$; same 
a

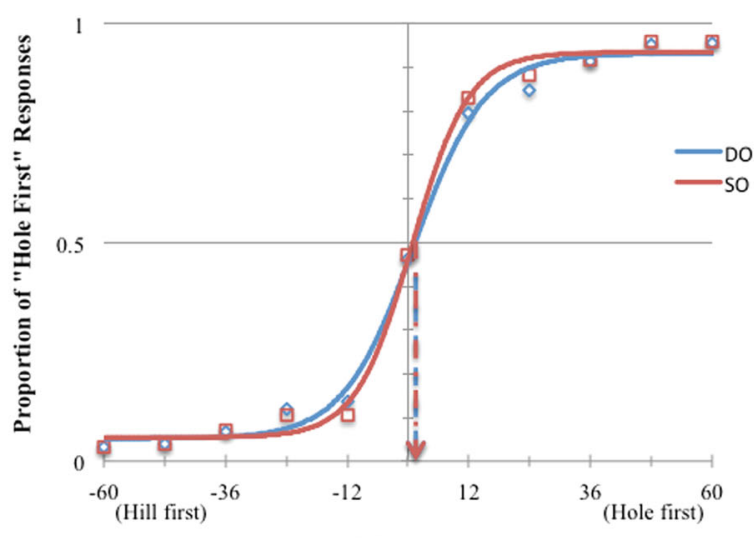

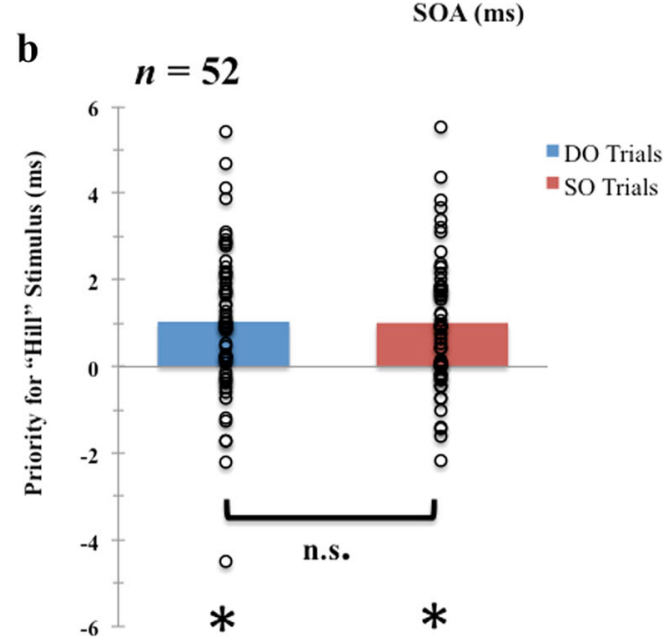

Fig. 3 Results from Experiment 2. (a) Aggregate TOJ functions for different-object (DO) and same-object (SO) trials. (b) Average PSS values for DO and SO trials. (c) PSS values by subjects, with colors

object: $t(51)=4.798, p<.001$, Cohen's $d=1.116$, scaled JZS Bayes factor $=1,355.179]$, but not different from one another (Fig. 3b).

Given that more participants were recruited for the study than was initially planned (the target was 20 participants per orientation), we ran a Monte Carlo simulation with 1,000 repetitions, subsampling 40 participants, 20 from the vertical group and 20 from the horizontal group. This revealed the same pattern of results as the full sample - namely, PSS scores significantly greater than 0 for different-object and sameobject trials in $>99.9 \%$ of simulations, and no significant difference between the PSS values on different-object and same object-trials in $>98 \%$ of simulations. It is important to note that having a larger sample increases the probability of making a Type I error (i.e., rejecting the null hypothesis when it is in fact true). In this case, we did not reject the null hypothesis, even with a rather large sample size.

These results strongly suggest that attentional selection, as measured by prior-entry effects, is not influenced by object structure in the absence of a spatial cue. Prior entry emerged in both same-object and different-object conditions, however to a similar extent in both trial types.

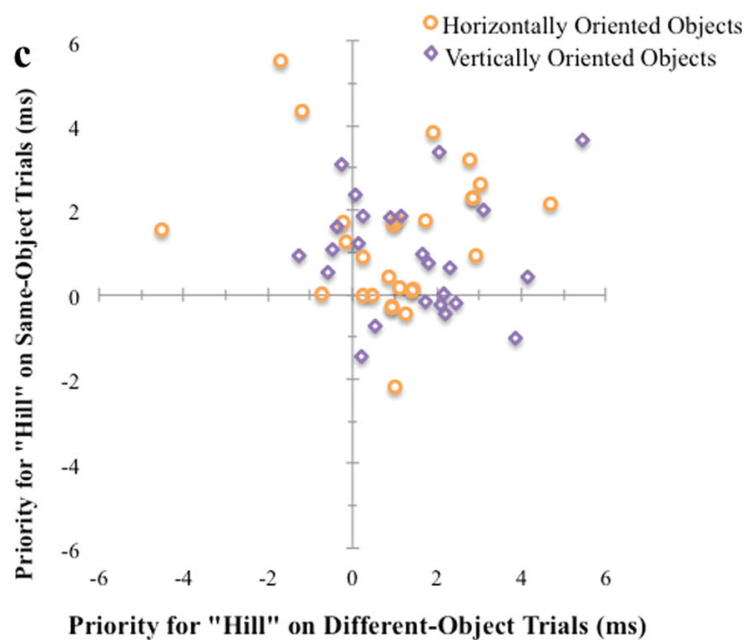

indicating whether the subject was presented with vertically oriented rectangles or horizontally oriented rectangles during the session

\section{Experiment 3}

The results of Experiment 2 demonstrated that, prior to the onset of the first target, attention is equally distributed across all four possible target locations, independent of objects. Given the absence of a spatial cue, the experimental design provided no incentive to attentionally bias any particular spatial location or an object. Because object-based representations were found to have no effects on prior entry, it remains unclear whether prior entry is simply not influenced by object representations, or whether object-based representations lack influence on prior entry without a spatial bias. If a spatial cue is in fact necessary for objects to influence the speed at which stimuli are perceived, no object-based difference in prior entry should be expected from the paradigm employed in Experiment 2, because of the lack of a spatial cue. We designed Experiment 3 to examine whether, in fact, objectbased representations can influence prior entry if attention is spatially biased.

An endogenous spatial bias is introduced by altering the relative frequencies of same-object and different-object trials. In this design, targets appearing on the same object were much 
more frequently, thereby biasing a particular object (i.e., cuing a particular object). To effectively bias a particular object, within each block of trials, targets appeared in only one of the two objects on same-object trials (e.g., left rectangle). This bias was reversed in another block of trials (i.e., now the right rectangle received most of the targets). The different-object targets were equally distributed among two different ends of the objects. This manipulation allowed us to focus endogenous attention on one of the two objects in preparation for the targets. The order of which objects contained stimuli on same-object trials (e.g., left then right, or right then left) was counterbalanced across participants. If object representations influence the distribution of attention in the presence of a spatial bias, then we predict that orienting attention to a particular object would result in a differential in prior entry effects on same-object (biased) than on different-object trials.

\section{Method}

Participants Forty-four undergraduates (31 women, 13 men) from George Washington University participated in Experiment 3 for course credit. All participants were naive to the purpose of the study and had normal or corrected-tonormal vision.

Apparatus, procedure, and design The apparatus, procedure, and design were the same as in Experiment 2, except for a few differences in the frequencies of certain trial types. First, same-object trials were four times more likely than different-object trials (176 same-object trials and 44 different-object trials per block). Additionally, on a single block, only one object was designated as the biased objectthat is, the object containing the stimuli on all same-object trials during that block. This served as an endogenous cue for the locations within the object, since it was advantageous for observers to allocate attention to locations in the object in which both stimuli appeared on the vast majority of trials (Geng \& Behrmann, 2002; Miller, 1987; Moore \& Egeth, 1998; Shaw, 1978). From block to block, the biased object in the same-object condition alternated. For example, with a vertical orientation of objects, if the first block had sameobject trials with targets in the rectangle on the left, the second block would have same-object trials with targets in the rectangle on the right, and they would alternate as such for the remaining blocks. For different-object trials, the two betweenobject target orientations were equally frequent. Therefore, $80 \%$ of trials were same-object trials, and the other $20 \%$ were different-object trials. Due to the increased frequency of sameobject relative to different-object trials, we increased the number of trials per block to 220 trials and decreased the number of blocks to four. Participants were not informed of the exact ratio of same-object to different-object trials; rather, they were simply told that targets could appear within either the same object or different objects on any given trial. Half of the participants were presented with horizontally oriented rectangles, whereas the other half was presented with vertically oriented rectangles. Trial order was randomized across all factors (first onset stimulus, same or different object, and SOA).

\section{Results and discussion}

As before, participants with overall accuracy below $70 \%$ or with no prior-entry effect for the "hill" were excluded. Participants with PSS values greater than two standard deviations from the mean for either same- or different-object trials were excluded (11 participants). As in the previous experiments, trials on which respondents chose a location that did not correspond to a target on the trial in question were excluded (1.30\% of responses).

The average accuracy was $86.71 \%$, with an average of $84.82 \%$ on different-object trials and of $92.09 \%$ on sameobject trials $[t(64)=4.15, p<.01]$, yielding greater accuracy for targets appearing within the more frequent, same-object configuration. An ANOVA on PSS values with Object (different vs. same object) as a within-subjects factor and Object Orientation (vertical vs. horizontal) as a between-subjects factor revealed a significant effect of object $[F(1,36)=8.065, p<$ $.01]$ and no significant effect of object orientation. The average PSS for different-object trials was calculated to be $0.838 \mathrm{~ms}$, which was not significantly different from 0 (Fig. 4a), whereas the average PSS for same-object trials was $1.193 \mathrm{~ms}$, which was significantly different from 0 $[t(32)=7.119, p<.01$, Cohen's $d=1.860$, scaled JZS Bayes factor $=318,903.3]$. As can be seen in Fig. $4 \mathrm{~b}$, the variances are more tightly distributed in the same-object condition, with mostly positive PSS values, whereas differentobject trials are more variable and span both positive and negative PSS values. Figure $4 \mathrm{c}$ shows similar distributions of PSS values for both horizontal and vertical orientations.

The results of this experiment strongly suggest that PSS can, in fact, be modulated by object representations, but that such modulation only occurs in the presence of a spatial attentional bias to a particular object.

\section{Experiment 4}

The results of Experiment 3 demonstrated that object-based influences on prior entry can, in fact, be elicited if an object representation is biased by endogenous attention. This conclusion was supported by the finding that prior-entry effects were modulated by object-based representations, if those representations were biased by endogenous attention. However, this difference may have been due to observers orienting attention according to space alone, since the target locations on same- 


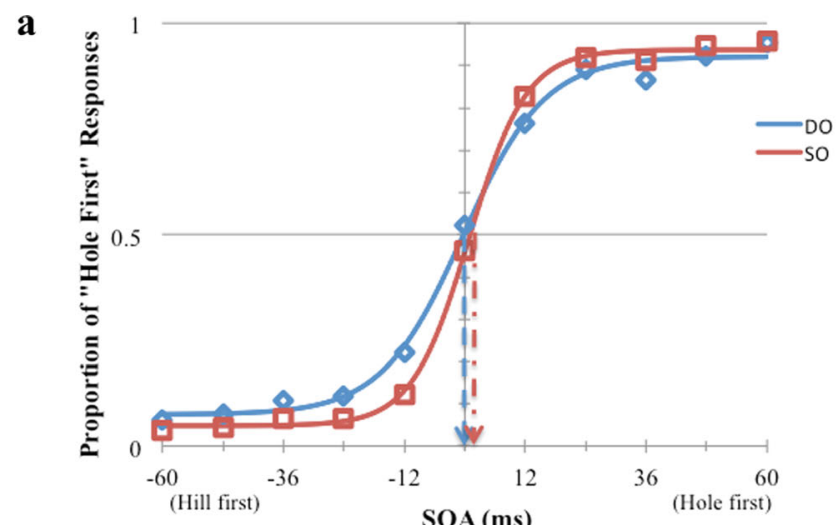

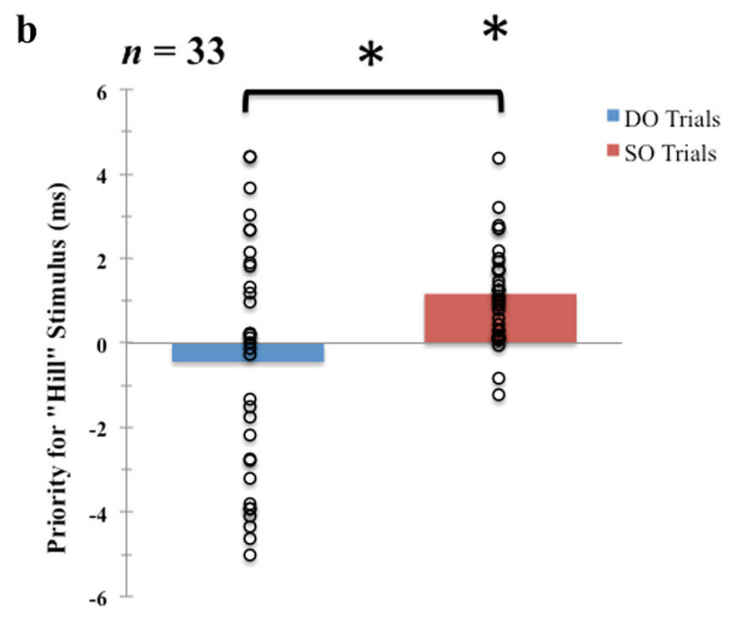

Fig. 4 Results from Experiment 3. (a) Aggregate TOJ functions for DO and SO trials. (b) Average PSS values for DO and SO trials. (c) PSS values by subjects, with colors indicating whether the subject was

object trials also corresponded to the spatial region occupied by the object. If the results in Experiment 3 were solely due to spatial orienting, the inverse results would be expected if different-object target locations were biased in the same way that same-object target locations had been biased. In other words, if spaced-based guidance alone determines the priorentry effect, a bias for different-object trials would result in prior entry in different-object but not in same-object trials. However, if a bias for different-object target locations did not result in a difference in prior entry between differentand same-object trials, this would indicate that the results from Experiment 3 represent an object-based effect. Furthermore, this would demonstrate that the contributions of space-based and object-based attentional guidance are further distinct than has previously been reported. Experiment 4 was designed to determine whether or not the apparent object-based effects observed in Experiment 3 were the result of object-based guidance, or simply due to space-based guidance (i.e., spatially binding locations that contain frequently co-occurring targets). Specifically, we altered the paradigm used in Experiment 3 such that instead of one within-object target location being biased per block, between-object locations would be biased per block.

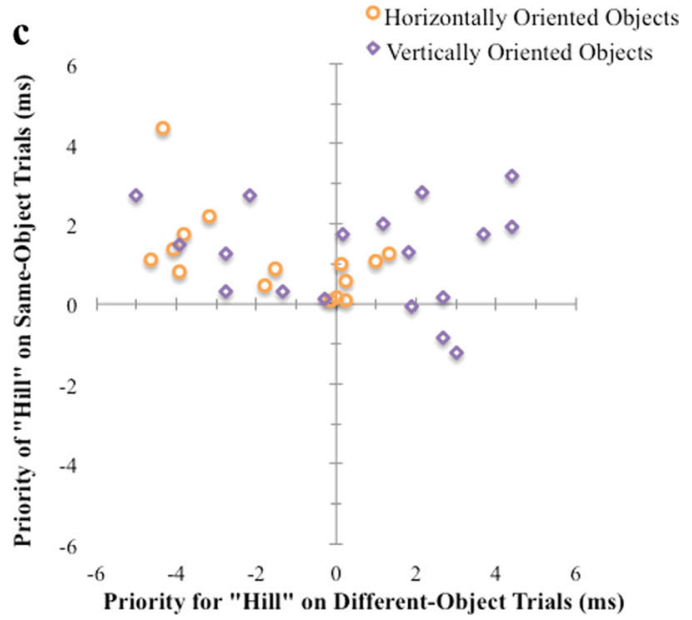

presented with vertically oriented rectangles or horizontally oriented rectangles during the session

\section{Method}

Participants Fifty-nine undergraduates (35 women, 24 men) from George Washington University participated in Experiment 4 for course credit. All participants were naive to the purpose of the study and had normal or corrected-to-normal vision.

Apparatus, procedure, and design The apparatus, procedure, and design were the same as in Experiment 3, except that different-object trials were four times as frequent as sameobject trials. Additionally, in a single block, only one betweenobject target orientation would occur on different-object displays. For example, with a vertical display, if the first block had different-object trials with targets on the top two locations, then the next block would have different-object trials with targets on the bottom locations. Notably, this was parallel to the design of Experiment 3, with the bias for same-object locations replaced by a bias for different-object locations.

\section{Results and discussion}

As in the previous experiments, participants with overall accuracy below $70 \%$ or with no prior-entry effect for the "hill" 
were excluded from the analysis. Participants who showed PSS values greater than two standard deviations from the mean for either same-object or different-object trials were also excluded (22 participants). Trials on which respondents chose a location that did not correspond to a target on the trial in question were excluded ( $<0.78 \%$ of responses). Half of participants in the vertical-rectangle group had a first block with different-object targets in the top two corners only, whereas the other half had a first block with different-object targets in the bottom two corners only. The parallel situation was true for those with horizontal-rectangle displays, such that half had a first block containing different-object trials with targets exclusively in the left corners, and the other half were given a first block with targets in the right corners only.

The average accuracy was $90.72 \%$, with $91.82 \%$ on different-object trials and $89.86 \%$ on same-object trials, which were not significantly different from one another. Unlike in Experiment 3, in which same-object targets were biased, there was no observable benefit for attending to the different-object trials. Figure 5a shows an aggregate TOJ function: sigmoids fitted to data collapsed across all observers and divided between same-object and different-object trials, with arrows indicating the aggregate PSS. Figure $5 \mathrm{~b}$ shows the average PSSs in different-object and same-object trials, with black circles indicating the individual values. Figure $5 \mathrm{c}$ shows the individual PSS values for different-object trials ( $x$-axis) and sameobject trials ( $y$-axis), with different colors indicating vertically oriented objects and horizontally oriented objects. The last plot shows a general trend toward positive PSS values on same-object trials, different-object trials, or both for the majority of participants. An ANOVA on PSS values with Object (different vs. same object) as a within-subjects factor and Object Orientation (vertical vs. horizontal) as a betweensubjects factor revealed no significant effect of object or object orientation. The average PSS value for different-object trials was $0.449 \mathrm{~ms}$, and that for same-object trials was $0.725 \mathrm{~ms}$, which were either significantly or marginally different from 0 [different object: $t(37)=2.116, p<.05$, Cohen's $d=0.70533$, scaled JZS Bayes factor $=1.277351$; same object: $t(36)=$ $2.467, p<.02$, Cohen's $d=0.82233$, scaled JZS Bayes factor $=2.49086$ ] (Fig. 5).

These results indicate that when targets appear in two between-object locations on the vast majority of trials on each block, both same-object and different-object trials elicit prior entry (similar to Exp. 1), though the effect is weaker. This suggests that the difference in prior entry between same- and different-object trials in Experiment 3 was due to object-based guidance, not to space-based orienting alone. In the present a

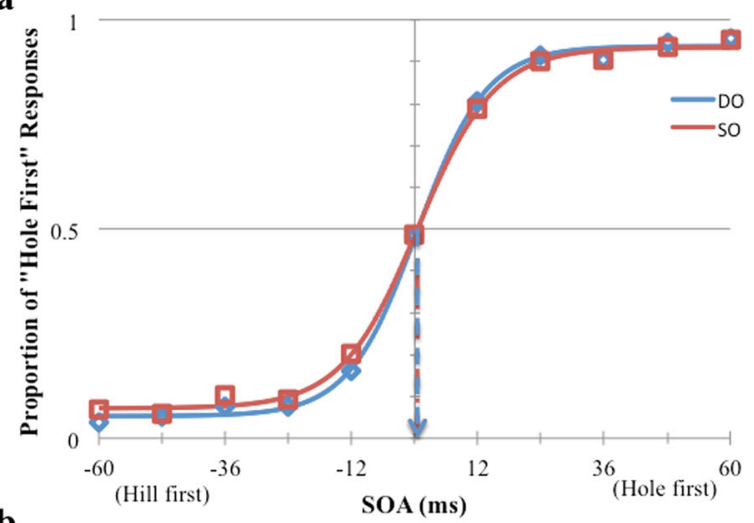

b

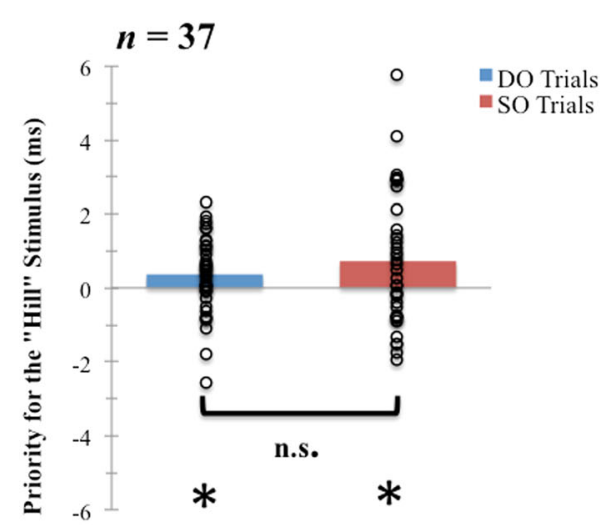

Fig. 5 Results from Experiment 4. (a) Aggregate TOJ functions for DO and SO trials. (b) Average PSS values for DO and SO trials. (c) PSS values by subjects, with colors indicating whether the subject was

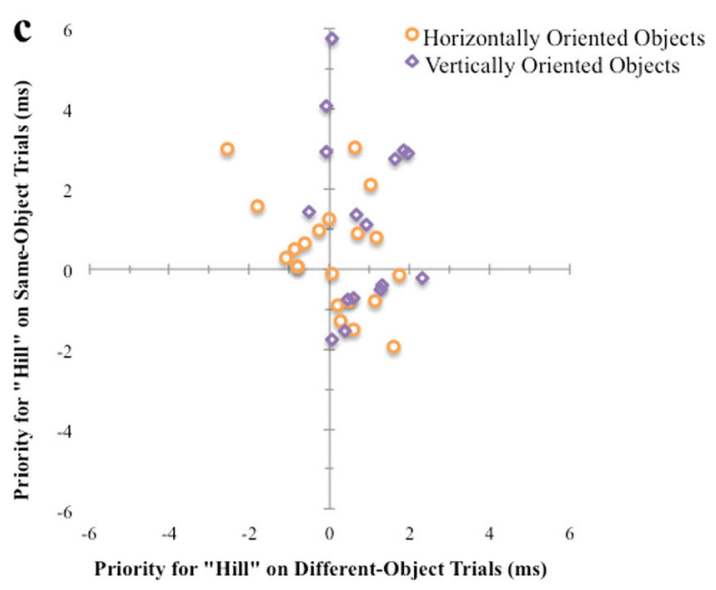

presented with vertically oriented rectangles or horizontally oriented rectangles during the session 
experiment, a bias for between-object locations, identical to the within-object bias in Experiment 3, did not elicit a difference in prior-entry effects between same- and different-object trials. Notably, the biased regions in Experiments 3 and 4 were equal in size and orientation, only differing in their relation to the object structure. The contribution of object representations to the distribution of attention, although constrained by a need for an initial allocation of attention to a spatial location on the object, remains distinct from space-based guidance itself.

\section{General discussion}

That object representations contribute to attentional selection has been demonstrated since the early 1980s (Duncan, 1981). As the contribution of visual objects to the distribution of attention continues to be characterized, it is important to dissociate object-based attention from other forms of attentional guidance. To date, all the research aiming to demonstrate influence of object-based representations to attentional guidance employed paradigms that, in one way or another, depended on endogenous or exogenous spatial cuing (Cepeda \& Kramer, 1999; Chen, 2012; Chen \& Cave, 2006; Richard et al., 2008; Shomstein, 2012). Indeed, it has recently been shown that spatial cues interact with top-down control settings to determine whether object-based effects are observed (Taylor, Rajsic, \& Pratt, 2016). This highlights the important of determining the isolated contribution of object representations in the absence of spatial cuing. Here, we aimed to elucidate whether space-based guidance is a necessary precursor for object-based attentional selection. To this end, we merged the traditional two-rectangle paradigm with a TOJ paradigm (for reviews, see Shore et al., 2001; Spence \& Parise, 2010; Stelmach \& Herdman, 1991; Ulrich, 1987). Our novel design of relying on TOJs allowed us, for the first time, to dissociate space- and object-based attentional selection, rather than examine an additional contribution exerted by objects above and beyond spatial selection.

Of note is that, although TOJs provide an elegant method to test attentional allocation without the use of a spatial cue, the size of the effect tends to be small. For example, most TOJ effects are on the order of 5-40 ms, similar to other attentional effects outside of spatial or endogenous cuing (for review, see Egeth \& Yantis, 1997). Understanding that we are dealing with modulations of small effects we took extra care in reporting multiple statistical tests that gave us confidence that even though the effects that we are reporting are small in magnitude, they are robust and replicable. To this end, for each reported $t$ test, we provide a corresponding Cohen's $d$, and a corresponding Bayes factor (Rouder et al., 2009). In all cases, consistent with the reported $t$ statistics, Cohen $d$ s were large, and Bayes factors provided decisive evidence against $H_{0}$ (computed using the BayesFactor package in R). In cases in which we reported a marginal significance, both Cohen's $d$ and the Bayes factor were in agreement with the $t$ statistic, warranting cautious interpretation.

The results obtained over a set of four experiments lead to several important new conclusions. First, we demonstrated that object-based representations do not guide attentional selection in the absence of spatial cues. This conclusion is supported by the finding that the observed prior-entry effects for salient "hill" stimulus (as measured by TOJ) was not modulated by whether the target stimuli shared the same or different object. Notably, the objects were starkly visible and were explicitly mentioned in the instructions to participants in Experiments 2, 3, and 4. However, there was no effect of objects on the prior-entry effect in Experiment 2, in which the target stimuli were equally likely to appear within or between objects. This finding indicates that object representations contribute to attentional guidance only when an object is spatially selected via an exogenous or endogenous cue, and therefore object representations contribute to attentional guidance only in tandem with spatial selection.

The second conclusion is that although object representations do not modulate prior-entry effects without spatial cues, objects can, in fact, modulate prior entry when spatial cues are present. In Experiments 3 and 4 we introduced an endogenous probability cue, such that targets were more likely to appear either on the same object (Exp. 3) or on different objects (Exp. 4), thus either biasing spatial locations that share an object representation or biasing spatial locations that are split between two objects. In Experiment 3, we show that prior entry can be modulated by object structure when attending to one object is advantageous for performance. Furthermore, this was distinctly an object-based effect, given that in Experiment 4, in which between-object locations were implicitly prioritized in a similar manner, different-object and same-object trials both elicited prior entry to similar degrees. Together, these findings indicate that locations within a visual object are ripe for enhanced attentional priority over other spatial regions, but will not guide attentional selection unless attention is already directed to the object or a spatial location within the object.

It should be considered that an object-based effect could have emerged in many different forms, including the presence of prior entry in both conditions but a difference in the magnitude between same-object prior entry and different-object prior entry, or the absence of prior entry in same-object trials but the presence of prior entry in different-object trials. The object-based effects in Experiment 3 emerged as the presence of prior entry in same-object trials and the absence of prior entry in different-object trials. This finding suggests a mechanism in which object-based selectively facilitates prior entry within an attended object at the cost of the extinction of prior entry in the unattended object. Still, the precise mechanism by which objects exert influence on prior entry remains unclear. Notably, prior entry indicates a bias for selecting one stimulus 
as appearing before a competing stimulus, specifically in conditions in which this is not the case. However, the effect of prior entry in only same-object trials in Experiment 3 was paired with greater accuracy in same-object trials than in different-object trials. This suggests that object influence on prior entry is not a result of less veridical temporal perception, but specifically the facilitation of prior-entry effects in an attended object and the extinction of prior entry in an unattended object.

The present pattern of findings also reveal the limitations of using exogenous cues, with or without corresponding endogenous cues, in determining how attention is allocated across the visual field (Chen, 2012; Chen \& Cave, 2008; Goldsmith \& Yeari, 2003; Law \& Abrams, 2002). Because cues have been the utterly dominant method of measuring attention for some 40 years, cue-based paradigms have become regarded as the gold standard for determining where, when, and how attention is distributed across space and objects. In many cases, using either exogenous and/or endogenous cues may be perfectly reasonable. But in the case of object-based attention, such cues have confounded space- and object-based attentional effects. It seems likely that in other situations, the unintended effects of using cues may have also masked the attentional behavior of interest.

Although our findings reveal that object-based effects did not emerge until attention was directed to spatial locations within a single object, it remains unclear to what extent object-based guidance of attention relies on other, nonspace-based forms of attentional selection. It is possible that in addition to relying on spatial representations, object-based guidance of attention also relies on other forms of representations, such as feature-based attention. Further investigation will be required to clarify the extent to which object-based attention is dependent on the selection of an object by various forms of attentional guidance, in addition to spatial attention. The outcome of such future experiments will not, however, diminish the main conclusion from the present study: Without cues or biases, objects do not guide attentional selection.

Author note This work was supported by grants from the National Science Foundation (BCS-1534823) and the National Institutes of Health (R21-EY021644) to S.S.

\section{References}

Arrington, C. M., Carr, T. H., Mayer, A. R., \& Rao, S. M. (2000). Neural mechanisms of visual attention: Object-based selection of a region in space. Journal of Cognitive Neuroscience, 12(Suppl. 2), 106-117. doi:10.1162/089892900563975

Becker, L., \& Egeth, H. (2000). Mixed reference frames for dynamic inhibition of return. Journal of Experimental Psychology Human Perception and Performance, 26, 1167-1177.

Carrasco, M. (2011). Visual attention: The past 25 years. Vision Research, 51, 1484-1525. doi:10.1016/j.visres.2011.04.012
Cepeda, N. J., \& Kramer, A. F. (1999). Strategic effects on object-based attentional selection. Acta Psychologica, 103, 1-19.

Chen, Z. (2012). Object-based attention: A tutorial review. Attention, Perception, \& Psychophysics, 74, 784-802. doi:10.3758/s13414012-0322-Z

Chen, Z., \& Cave, K. R. (2006). Reinstating object-based attention under positional certainty: The importance of subjective parsing. Perception \& Psychophysics, 68, 992-1003. doi:10.3758/BF03193360

Chen, Z., \& Cave, K. R. (2008). Object-based attention with endogenous cuing and positional certainty. Perception \& Psychophysics, 70, 1435-1443. doi:10.3758/PP.70.8.1435

Driver, J., \& Baylis, G. C. (1991). Target-distractor separation and feature integration in visual attention to letters. Acta Psychologica, 76, 101-119.

Duncan, J. (1981). Directing attention in the visual field. Perception \& Psychophysics, 30, 90-93.

Duncan, J. (1984). Selective attention and the organization of visual information. Journal of Experimental Psychology: General, 113, 501517. doi:10.1037/0096-3445.113.4.501

Egeth, H. E., \& Yantis, S. (1997). Visual attention: Control, representation, and time course. Annual Review of Psychology, 48, 269-297. doi:10.1146/annurev.psych.48.1.269

Egly, R., Driver, J., \& Rafal, R. D. (1994). Shifting visual attention between objects and locations: Evidence from normal and parietal lesion subjects. Journal of Experimental Psychology: General, 123, 161-177. doi:10.1037/0096-3445.123.2.161

Eriksen, B. A., \& Eriksen, C. W. (1974). Effects of noise letters upon the identification of a target letter in a nonsearch task. Perception \& Psychophysics, 16, 143-149. doi:10.3758/BF03203267

Fiebelkorn, I. C., Saalmann, Y. B., \& Kastner, S. (2013). Rhythmic sampling within and between objects despite sustained attention at a cued location. Current Biology, 23, 2553-2558. doi:10.1016/j. cub.2013.10.063

Geng, J. J., \& Behrmann, M. (2002). Probability cuing of target location facilitates visual search implicitly in normal participants and patients with hemispatial neglect. Psychological Science, 13, 520-525.

Goldsmith, M., \& Yeari, M. (2003). Modulation of object-based attention by spatial focus under endogenous and exogenous orienting. Journal of Experimental Psychology: Human Perception and Performance, 29, 897-918. doi:10.1037/0096-1523.29.5.897

Hollingworth, A., Maxcey-Richard, A. M., \& Vecera, S. P. (2012). The spatial distribution of attention within and across objects. Journal of Experimental Psychology: Human Perception and Performance, 38, 135-151. doi:10.1037/a0024463

Kramer, A. F., \& Jacobson, A. (1991). Perceptual organization and focused attention: The role of objects and proximity in visual processing. Perception \& Psychophysics, 50, 267-284. doi:10.3758 /BF03206750

Kravitz, D. J., \& Behrmann, M. (2011). Space-, object-, and feature-based attention interact to organize visual scenes. Attention, Perception, \& Psychophysics, 73, 2434-2447. doi:10.3758/s13414-011-0201-z

Lavie, N., \& Driver, J. (1996). On the spatial extent of attention in objectbased visual selection. Perception \& Psychophysics, 58, 12381251. doi:10.3758/BF03207556

Law, M. B., \& Abrams, R. A. (2002). Object-based selection within and beyond the focus of spatial attention. Perception \& Psychophysics, 64, 1017-1027. doi:10.3758/BF03194753

Miller, J. (1987). Priming is not necessary for selective-attention failures: Semantic effects of unattanded, unprimed letters. Perception \& Psychophysics, 41, 419-434. doi:10.3758/BF03203035

Moore, C. M., \& Egeth, H. (1998). How does feature-based attention affect visual processing? Journal of Experimental Psychology: Human Perception and Performance, 24, 1296-1310. doi:10.1037/0096-1523.24.4.1296

Posner, M. I. (1980). Orienting of attention. Quarterly Journal of Experimental Psychology, 32, 3-25. doi:10.1080/00335558008248231 
Posner, M. I., Walker, J. A., Friedrich, F. J., \& Rafal, R. D. (1984). Effects of parietal injury on covert orienting of attention. Journal of Neuroscience, 4, 1863-1874.

Pratt, J., \& Sekuler, A. B. (2001). The effects of occlusion and past experience on the allocation of object-based attention. Psychonomic Bulletin \& Review, 8, 721-727. doi:10.3758/BF03196209

Richard, A. M., Lee, H., \& Vecera, S. P. (2008). Attentional spreading in object-based attention. Journal of Experimental Psychology: Human Perception and Performance, 34, 842-853. doi:10.1037/00961523.34.4.842

Rouder, J. N., Speckman, P. L., Sun, D., Morey, R. D., \& Iverson, G. (2009). Bayesian $t$ tests for accepting and rejecting the null hypothesis. Psychonomic Bulletin \& Review, 16, 225-237. doi:10.3758/PBR.16.2.225

Scholl, B. J. (2001). Objects and attention: The state of the art. Cognition, 80, 1-46. doi:10.1016/S0010-0277(00)00152-9

Serences, J. T., \& Yantis, S. (2006). Selective visual attention and perceptual coherence. Trends in Cognitive Sciences, 10, 38-45. doi:10.1016/j.tics.2005.11.008

Shaw, M. L. (1978). A capacity allocation model for reaction time. Journal of Experimental Psychology: Human Perception and Performance, 4, 586-598.

Shomstein, S. (2012). Object-based attention: Strategy versus automaticity. Wiley Interdisciplinary Reviews: Cognitive Science, 3, 163-169. doi: $10.1002 /$ wcs. 1162

Shomstein, S., \& Yantis, S. (2002). Object-based attention: Sensory modulation or priority setting? Perception \& Psychophysics, 64, 41-51. doi:10.3758/BF03194556

Shomstein, S., \& Yantis, S. (2004). Configural and contextual prioritization in object-based attention. Psychonomic Bulletin \& Review, 11, 247-253. doi:10.3758/BF03196566

Shore, D. I., Spence, C., \& Klein, R. M. (2001). Visual prior entry. Psychological Science, 12, 205-212. doi:10.1111/1467-9280.00337
Spence, C., \& Parise, C. (2010). Prior-entry: A review. Consciousness and Cognition, 19, 364-379. doi:10.1016/j.concog.2009.12.001

Spence, C., Shore, D. I., \& Klein, R. M. (2001). Multisensory prior entry. Journal of Experimental Psychology: General, 130, 799-832. doi:10.1037/0096-3445.130.4.799

Stelmach, L. B., \& Herdman, C. M. (1991). Directed attention and perception of temporal order. Journal of Experimental Psychology: Human Perception and Performance, 17, 539-550. doi:10.1037/0096-1523.17.2.539

Taylor, J. E., Rajsic, J., \& Pratt, J. (2016). Object-based selection is contingent on attentional control settings. Attention, Perception, \& Psychophysics, 78, 988-995. doi:10.3758/s13414-016-1074-y

Ulrich, R. (1987). Threshold models of temporal-order judgments evaluated by a ternary response task. Perception \& Psychophysics, 42, 224-239. doi:10.3758/BF03203074

Vecera, S. P. (1997). Grouped arrays versus object-based representations: Reply to Kramer et al. (1997). Journal of Experimental Psychology: General, 126, 14-18.

Watson, S. E., \& Kramer, A. F. (1999). Object-based visual selective attention and perceptual organization. Perception \& Psychophysics, 61, 31-49. doi:10.3758/BF03211947

West, G. L., Anderson, A. K., Bedwell, J. S., \& Pratt, J. (2010). Red diffuse light suppresses the accelerated perception of fear. Psychological Science, 21, 992-999. doi:10.1177/0956797610371966

West, G. L., Anderson, A. A. K., \& Pratt, J. (2009). Motivationally significant stimuli show visual prior entry: Evidence for attentional capture. Journal of Experimental Psychology: Human Perception and Performance, 35, 10321042. doi: $10.1037 / \mathrm{a} 0014493$

West, G. L., Pratt, J., \& Peterson, M. A. (2013). Attention is biased to near surfaces. Psychonomic Bulletin \& Review, 20, 1213-1220. doi:10.3758/s13423-013-0455-7 